\title{
Anurofauna da bacia do Rio Tijuco, Minas Gerais, Brasil e sua relação com taxocenoses de anfíbios do Cerrado e suas transições
}

\author{
Carlos E. Conte ${ }^{1,2}$, Daniele R. da Silva ${ }^{3}$ \& André P. Rodrigues ${ }^{4}$
}

\author{
1. Departamento de Zoologia, Universidade Federal do Paraná, Caixa Postal 19020, 81531-980 Curitiba, PR, Brasil. Bolsista PRODOC/CAPES \\ 2. Instituto Neotropical: Pesquisa e Conservação, Caixa Postal 19009, 81531-980 Curitiba, PR, Brasil. \\ 3. Programa de Pós-Graduação em Ecologia e Conservação de Recursos Naturais, Universidade Federal de Uberlândia, 38408-100, Uberlândia, MG, Brasil. \\ 4. Instituto Brasileiro do Meio Ambiente e Recursos Naturais Renováveis - IBAMA, 47804-140, Barreiras, BA, Brasil.
}

\begin{abstract}
Anurofauna from the Tijuco River basin, state of Minas Gerais, Brazil and its relationship with assemblages of amphibians from Cerrado and their transitions. The suppression of natural landscapes has decimated populations and/or reduced the area of occurrence of many anuran amphibian species. This is the scenario of Cerrado, given its great economic potential and the decay speed through human activities. For this reason, species inventories are extremely needed in such regions, where the dispersion activity and reproduction might be beset depending on the degree of environmental alteration. This study aims to determine the richness and the diversity of reproductive modes of the anurofauna from the Tijuco River basin and to compare the composition of the assemblage from such region with assemblages of amphibians previously studied from other regions within the biomes Cerrado and Mesophytic Semideciduous Forest. The study was carried out along the Tijuco River basin in and approximate extension of $80 \mathrm{~km}$. We conduct four sampling methods where 40 species of anurans belonging to six families were registered. This corresponds to $20 \%$ of the species of anurans known to Cerrado. The landscape conditions favor species more tolerant and adapted to dry weather, which reflects an assemblage with lower proportions of reproductive modes and higher occurrence of generalized modes, dependent of water or adapted against desiccation. The comparison with 15 different localities revealed that the anurofauna from Cerrado is unique, with low taxonomic similarity to the adjacent ecosystems. The diversity of habitats and the expressive richness of species make the Tijuco River basin very important for the conservation of the anurofauna from the biome Cerrado.
\end{abstract}

KEYWORDS. Anura, distribution, modified landscape, community ecology.

RESUMO. A supressão de paisagens naturais tem dizimado populações e/ou reduzido a área de ocorrência de muitas espécies de anuros, caso do Cerrado, dado o grande potencial econômico e velocidade de descaracterização por atividades humanas. Por este motivo, inventários de espécies são extremamente necessários nessas regiões que dependendo do grau de alteração ambiental podem prejudicar as atividades de dispersão e reprodução dos anuros. Este estudo tem por objetivo determinar a riqueza e a diversidade de modos reprodutivos da anurofauna da bacia do Rio Tijuco e comparar a composição da taxocenose dessa região com taxocenoses de anfíbios de outras regiões do bioma Cerrado e da floresta estacional semidecidual anteriormente estudadas. O estudo foi desenvolvido ao longo da bacia do Rio Tijuco numa extensão aproximada de 80 $\mathrm{km}$. Através de quatro métodos de amostragem, foram registradas 40 espécies de anuros pertencentes a seis famílias, o que corresponde a $20 \%$ das espécies de anuros registradas para o Cerrado. As condições da paisagem favorecem espécies mais tolerantes e adaptadas ao clima mais seco, o que reflete numa taxocenose que possui baixa proporção de modos reprodutivos e uma elevada representatividade de modos generalizados, dependentes de água ou com adaptações contra dessecação. Em comparação com outras 15 localidades verificou-se que a anurofauna do Cerrado é típica, com baixa similaridade taxonômica com ecossistemas adjacentes. A diversidade de habitats e a expressiva riqueza de espécies tornam a bacia do Rio Tijuco de grande importância para a conservação da anurofauna do bioma Cerrado.

PALAVRAS-CHAVE. Anuros, distribuição, paisagem modificada, ecologia de comunidades.

O Cerrado é a segunda maior região biogeográfica da América do Sul, ocupando cerca de $25 \%$ do território brasileiro (EITEN, 1972; Coutinho, 1978; KLinK et al., 2002). Por sua vasta distribuição, exibe um mosaico de fitofisionomias (KLINK et al., 2002) que abrigam uma elevada diversidade de anfíbios, sendo conhecidas cerca de 209 espécies, aproximadamente $22 \%$ do total encontrado no Brasil (VAldujo et al., 2012).

Por infortúnio, informações básicas sobre as espécies de anfíbios desse bioma são praticamente inexistentes frente à elevada complexidade de ecossistemas que compõe o Cerrado (HADDAD et al., 1988; Rocha, 1994; Strüssmann, 2000; Colli et al., 2002; Diniz-Filho et al., 2004a,b; ETEROVICK \& SAZima, 2004; Silveira, 2006; GiaretTa et al., 2008), havendo muitas localidades não amostradas ou com informações insuficientes sobre as espécies (CoLli et al., 2002; Bastos, 2007; VAldujo et al., 2012). Como agravante, a situação da conservação do Cerrado é particularmente crítica e, consequentemente da biota associada ao Bioma, dado o grande potencial econômico e velocidade de descaracterização por atividades humanas (KLINK \& MACHADO, 2005). Muito se debate sobre declínio populacional e possíveis extinções de anuros ao redor do mundo (PHILlips, 1990; REASER, 2000), sendo a principal causa associada à destruição do habitat, que pode ser relacionada à poluição das águas e à contaminação por pesticidas (Young et al., 2004).

Segundo Myers et al. (2000), trabalhos sobre diversidade são particularmente importantes e urgentes em áreas de alta biodiversidade afetadas pela ação humana, situação em que se enquadra a Bacia do Rio Tijuco, onde parte da região está entre as áreas prioritárias para conservação na categoria extremamente alta (MMA, 2002). Assim, o presente estudo tem como objetivo determinar a riqueza e a diversidade de modos reprodutivos da anurofauna da bacia do Rio Tijuco, localizado no triângulo mineiro, Minas Gerais, Brasil e comparar a composição de espécies dessa região com taxocenoses de anfíbios de outras regiões do bioma Cerrado e da Floresta Estacional Semidecidual anteriormente estudadas. 


\section{MATERIAL E MÉTODOS}

Área de estudo. A bacia do Rio Tijuco ocupa uma área de aproximadamente $27 \%$ do Triângulo Mineiro (SANTOS \& BACCARo, 2004) e sua extensão abrange os municípios de Uberlândia, Uberaba, Veríssimo, Ituiutaba, Prata, Monte Alegre de Minas e Campina Verde, tendo como principais afluentes os rios Prata, Babilônia, Cabaçal, Douradinho, Panga e Estiva (SANTOS \& BACCARO, 2004). A região - apesar da intensa alteração ambiental causada pela expansão da agricultura e pecuária (GoBBi et al., 2008) -, ainda conta com trechos remanescentes com fitofisionomias típicas do Cerrado latu sensu (SANtos \& Baccaro, 2004), seguindo a caracterização de EITEN (1972), cujas principais fisionomias reconhecidas são o cerradão, cerrado sensu stricto, campo cerrado, campo sujo e campo limpo.

O estudo foi desenvolvido nos municípios de Monte Alegre de Minas e Ituiutaba, ao longo da bacia do Rio Tijuco, em oito pontos de coleta numa extensão aproximada de $80 \mathrm{~km}$ (Fig. 1).

O clima predominante é o tropical, com duas estações bem definidas, uma quente e úmida, de outubro a março e outra menos quente e seca, de abril a setembro (SANTOS \& BACCARO, 2004). Os índices pluviométricos variam de 1500 a $2000 \mathrm{~mm}$, distribuídos irregularmente durante todo o ano, com maior volume (ca. de 50\%) concentrados no verão (SANTOS \& BACCARO, 2004).

Coleta de dados. Foram realizadas coletas mensais com duração de três dias cada, entre os meses de agosto de 2005 e fevereiro de 2006. A cada visita foram empregados quatro métodos para amostragem dos anuros: 1) levantamento em sítio de reprodução (sensu SCOTT JR. \& WoODWARD, 1994) no qual o perímetro de cada corpo d'água foi percorrido lentamente, sendo registradas as espécies visualizadas bem como machos em atividade de vocalização; 2) transecção por busca auditiva e visual (adaptado de ZIMMERMAN, 1994) efetuado em um trecho de $1,5 \mathrm{~km}$ no interior da floresta, que foi percorrido lentamente, em linha reta, sendo registradas as espécies cujos machos vocalizam distantes de corpos d'água; 3 ) busca ativa, que consistiu na procura dos anfíbios embaixo de troncos, pedras e demais refúgios encontrados no entorno de cada corpo d'água e no interior de floresta, com um esforço de procura 62 horas/pessoa em cada local. Além disso, duas fases adicionais de 12 dias foram realizadas nos meses de janeiro de 2007 e março de 2008, utilizando os mesmos métodos com a adição de 4) armadilhas de interceptação e queda (adaptado de CoRN, 1994), que consiste de recipientes enterrados com a abertura ao nível do solo (pitfalls) e interligados por cercas-guia (drift-fences). Para distribuição das armadilhas, foram previamente escolhidos seis pontos, cada um próximo à margem do leito do Rio Tijuco. Em cada linha de 40 $\mathrm{m}$ foram distribuídos equidistantemente cinco baldes de $60 \mathrm{~L}$.

Construção da base de dados para comparação da anurofauna. A composição das espécies do Rio Tijuco foi comparada com a de outras 15 localidades, que foram separadas em quatro ecossistemas: Cerrado (CER), Floresta Estacional Semidecidual (FES), transição entre o Cerrado e a Caatinga (CEC) e transição entre o Cerrado e a Floresta Estacional Semidecidual (CEF) (Tab. I). A base de dados foi compilada da literatura (Tab. I), sendo

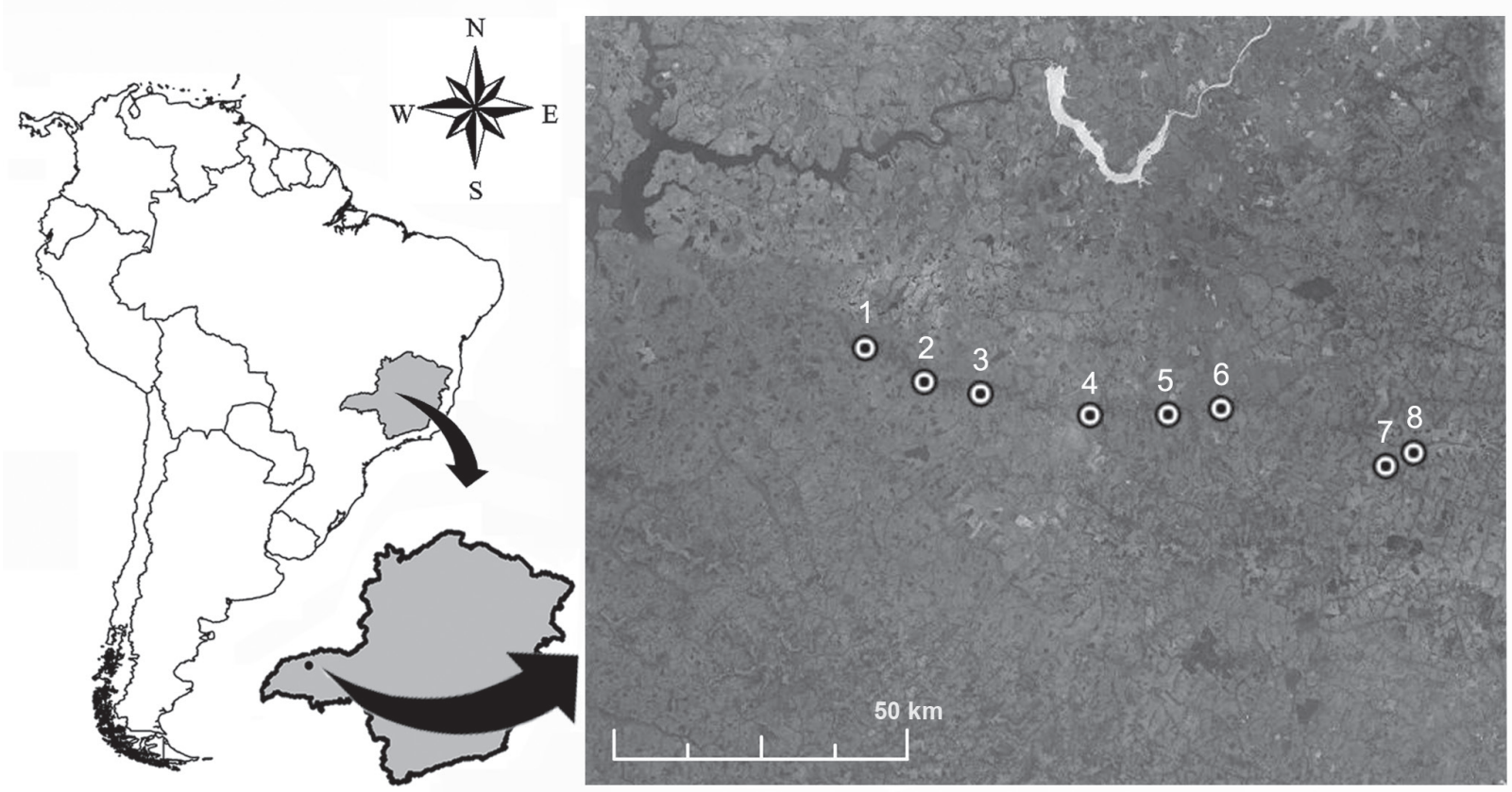

Fig. 1. Mapa de localização dos pontos amostrados na bacia do rio Tijuco, região do Triângulo Mineiro, Minas Gerais, Brasil. Coordenadas dos pontos de coleta: (1) $18^{\circ} 51^{\prime} 30,24^{\prime \prime} \mathrm{S}, 49^{\circ} 41^{\prime} 35,80^{\prime \prime} \mathrm{W}$; (2) 18 $58^{\circ} 59,44^{\prime \prime} \mathrm{S}, 49^{\circ} 20^{\prime} 21,83^{\prime \prime} \mathrm{W}$; (3) $18^{\circ} 55^{\prime} 54,58^{\prime \prime} \mathrm{S}, 49^{\circ} 34^{\prime} 53,89^{\prime \prime} \mathrm{W}$; (4)

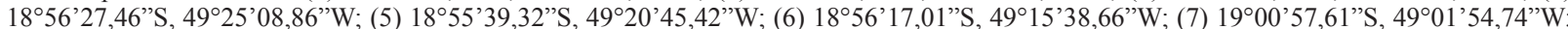
(8) $19^{\circ} 00^{\prime} 02,71^{\prime \prime S}, 49^{\circ} 04^{\prime} 32,28^{\prime \prime} \mathrm{W}$ (imagem adaptada do Google Earth). 
considerados apenas os registros de espécies obtidos em estudos com amostragens sequenciais durante os meses quentes e úmidos do ano, numa tentativa de trabalhar com listas de espécies mais acuradas, devido à sazonalidade na ocorrência temporal dos anuros (Wells, 2007). Para evitar a interferência de problemas taxonômicos, foram excluídas das análises espécies não identificadas (affinis e confers) e as espécies do gênero Pseudis, devido a possíveis erros na identificação. Deste modo, foi gerada uma lista com 119 espécies em que $20 \%(n=24$ espécies $)$ não foram consideradas. Os resultados foram inseridos em uma matriz de incidência (presença x ausência) de espécies por localidade. Modos reprodutivos são baseados em HADDAD \& PRADo (2005) e a taxonomia seguiu Frost (2013).

Análises estatísticas. Para verificar se há diferença entre as taxocenoses de cada ecossistema foi conduzida uma Análise de Similaridade (ANOSIM). Esta análise testa a diferença entre dois ou mais grupos de unidades amostrais a partir de uma matriz de distâncias transformada em ranks (ClaRKE, 1993; LEGENDRE \& LegENDRE, 1998). Aqui, as unidades amostrais correspondem as localidades e a variável classificadora (grupos) as formações vegetais, utilizando como medida de distância o índice de Bray-Curtis. Para representar graficamente as diferenças entre os grupos, foi utilizada análise de escalonamento multidimensional não métrico (NMDS: ClaRKE \& WARWICK, 1994) utilizando como medida o índice de similaridade de Jaccard. Para verificar a contribuição relativa das espécies para a formação dos agrupamentos detectados na ANOSIM, foi usada uma análise de percentual de dissimilaridade (SIMPER: ClARKE, 1993). Considerando a alta riqueza, os resultados são baseados nas espécies que mais contribuíram utilizando como critério os valores de até $20 \%$ de contribuição cumulativa. Para verificar se a distribuição das taxocenoses analisadas é um efeito da distância geográfica, foi realizado o teste de correlação de Mantel (Manly, 2008). Esse teste determina a significância de correlação entre matrizes de similaridade ou de distância a partir de dados multidimensionais e o posterior cálculo utilizando permutações de Monte Carlo (Manty, 2008). As análises foram realizadas com os softwares PAST v.1.75b (HAMMER et al., 2001), Primer (Clark \& Gorley, 2006) e R (R Development Core Team, 2012).

\section{RESULTADOS}

Foram registradas 40 espécies de anuros pertencentesaseisfamílias:Bufonidae(1), Craugastoridae (1), Hylidae (15), Leiuperidae (7), Leptodactylidae (17), Microhylidae (4) e Odontophrynidae (2). Para atividade de vocalização e/ou reprodução, a maioria das espécies foi encontrada em açudes e poças temporárias (Tab. II). A proporção de modos reprodutivos foi de 0,22 ( 9 modos/40 espécies), sendo o modo reprodutivo 01 o mais comum na área estudada, utilizado por 22 espécies (58\% do total) e com grande representatividade em Hylidae (Tab. II).

A anurofauna do Bioma Cerrado é típica, e apresenta baixa similaridade taxonômica com os ecossistemas adjacentes $(\mathrm{R}=0,47, \mathrm{P}=0,002$; Tab. III, Fig. 2). A composição e distribuição das taxocenoses analisadas são reguladas pela distância geográfica $\left(\mathrm{r}^{2}=\right.$ $0,72 ; \mathrm{p}<0,0001)$, com uma dissimilaridade média de

Tab. I. Lista de localidades com inventários de anfíbios (EE, estação ecológica; FLOE, Floresta Estadual; FLONA, Floresta Nacional; MUN, município; PARNA, Parque Nacional. EA, esforço amostral: dia/meses. Métodos de inventário: 1, armadilhas de interceptação e queda; 2, amostragem em sítio de reprodução; 3 , busca ativa; 4 , transecção por busca visual e aural. Spp/nie, número de espécies registradas/número de espécies não identificadas em nível específico).

\begin{tabular}{|c|c|c|c|c|c|}
\hline Localidade & Sigla & EA & Método & Spp/nie & Referência \\
\hline \multicolumn{6}{|l|}{ Cerrado } \\
\hline Bacia do Rio Tijuco, MG & BRT & $20 / 2$ & $1,2,3,4$ & $41 / 05$ & Presente estudo \\
\hline FLONA de Silvânia, GO & FNS & $--/ 60$ & 1,2 & $29 / 02$ & BASTOS et al., 2003 \\
\hline PARNA de Emas, GO & EMA & $--/ 16$ & 2 & $25 / 04$ & KoOP et al., 2010 \\
\hline EE de Itirapina, SP & EEI & $--/ 43$ & 1,2 & $28 / 03$ & BRASILEIRO et al., 2005 \\
\hline EE de Assis, SP & EEA & $--/ 25$ & 2 & $23 / 03$ & Ribeiro-Junior \& BERTOLUCi, 2009 \\
\hline MUN de Morrinhos, GO & MOR & $36 / 12$ & 2 & $26 / 04$ & Borges \& Juliano, 2007 \\
\hline MUN de Niquelândia, GO & NIQ & $36 / 06$ & 2 & $34 / 05$ & NoMURA et al., 2012 \\
\hline EE Serra Geral do Tocantins, TO & JAL & $20 / 02$ & 1 & $35 / 01$ & VALDUJO et al., 2011 \\
\hline \multicolumn{6}{|c|}{ Transição entre o Cerrado e a Floresta Estacional Semidecidual } \\
\hline Fazenda Rio Claro, SP & OES & $--/ 24$ & 2 & 40/-- & MAFFEI et al., 2011 \\
\hline \multicolumn{6}{|l|}{ Transição entre o Cerrado e a Caatinga } \\
\hline MUN de São Desidério, BA & SDE & $13 / 03$ & 1 & $32 / 03$ & VALDUJO et al., 2009 \\
\hline \multicolumn{6}{|l|}{ Floresta Estacional Semidecidual } \\
\hline Mata São José, SP & MSJ & $--/ 12$ & 2 & $24 / 05$ & ZINA et al., 2007 \\
\hline MUN de Guararapes, SP & GUA & $--/ 26$ & 2 & $26 / 01$ & Bernarde \& KoKubum, 1999 \\
\hline MUN de Nova Itapirema, SP & $\mathrm{NOV}$ & $--/ 15$ & 2 & $27 / 04$ & VASCONCELOS \& RosSA-FERES, 2005 \\
\hline EE dos Caetetus, SP & CAE & $--/ 35$ & 1,2 & $34 / 05$ & BRASSALOTI et al., 2010 \\
\hline MUN de Icém, SP & ICE & $--/ 12$ & 2 & 26/-- & SiLVA et al., 2012 \\
\hline FLOE Edmundo N. de Andrade & MRC & $--/ 17$ & 2 & $21 / 04$ & ToLEDO et al., 2003 \\
\hline
\end{tabular}


Tab. II. Lista das espécies de anfíbios anuros registrados ao longo da bacia do rio Tijuco, região do Triângulo Mineiro, Minas Gerais, Brasil (Método de amostragem: 1, levantamento em sítio de reprodução; 2, transecção por busca auditiva e visual; 3, busca ativa; 4, armadilhas de interceptação e queda. Fisionomias: 1, mata de galeria e/ou remanescentes de Floresta Estacional; 2, campo cerrado; 3, campo sujo; 4, campo limpo; 5, veredas; 6, áreas alteradas com pastagens. Ambientes utilizados para atividade de reprodução (AR): 1, serapilheira; 2, poças temporárias; 3, açudes; 4, riachos; 5, áreas com pouca quantidade de água (lâmina d'água); 6, veredas. Modo reprodutivo (MR) (sensu HADDAD \& Prado, 2005): 1, ovos e larvas exotróficas em hábitats aquáticos lênticos; 2, ovos e larvas exotróficas em hábitats aquáticos lóticos; 4, ovos e estágios iniciais de desenvolvimento em "piscinas" naturais ou construídas, subsequentes ao enchimento, larvas exotróficas em córregos ou poças; 11, ovos depositados em ninhos de espuma flutuante larvas exotróficas em poças; 13, ovos depositados em ninhos de espuma depositados sobre água acumulada em uma depressão construída, larvas exotróficas em poças; 23, desenvolvimento direto de ovos terrestres; 29 , ninhos de espuma com ovos e estágios iniciais de desenvolvimento em piscinas e após transbordamento, girinos exotróficos em poças ou riachos; 30, ninho de espuma com ovos e estágios iniciais de desenvolvimento em ninhos construídos subterrâneos, subsequentes ao enchimento, larvas exotróficas em poças).

\begin{tabular}{|c|c|c|c|c|}
\hline Táxon & Método de amostragem & Fisionomia & AR & MR \\
\hline \multicolumn{5}{|l|}{ Bufonidae } \\
\hline Rhinella schneideri (Werner, 1894) & 1,3 & $2,3,4,6$ & $2,3,4$ & 1 \\
\hline \multicolumn{5}{|l|}{ Craugastoridae } \\
\hline Barycholos ternetzi (Miranda-Ribeiro, 1937) & 2 & 1 & 1 & 23 \\
\hline \multicolumn{5}{|l|}{ Hylidae } \\
\hline Dendropsophus cruzi (Pombal \& Bastos, 1998) & 1 & $1,2,3$ & 2 & 1 \\
\hline D. jimi (Napoli \& Caramaschi, 1999) & 1,3 & & 2,3 & 1 \\
\hline D. minutus (Peters, 1872) & 1,3 & $2,3,4,6$ & 2,3 & 1 \\
\hline D. nanus (Boulenger, 1889) & 1,3 & $2,3,4,6$ & 2,3 & 1 \\
\hline D. rubicundulus (Reinhardt \& Lütken, 1862) & 1,3 & $2,3,4,6$ & 2,3 & 1 \\
\hline Dendropsophus cf. melanargyreus & 1,3 & 1,2 & 2 & 1 \\
\hline Hypsiboas albopunctatus (Spix, 1824) & 1,3 & $2,3,4,6$ & 2,3 & 1 \\
\hline H. lundii (Burmeister, 1856) & 1,3 & 1 & 4 & 4 \\
\hline H. paranaiba Carvalho \& Giaretta, 2010 & 1,3 & 1,2 & 4,5 & 1 \\
\hline H. raniceps Cope, 1862 & 1,3 & $2,3,4,6$ & 2,3 & 1 \\
\hline Phyllomedusa azurea Cope, 1862 & 1,3 & $2,3,4,6$ & 2,3 & 24 \\
\hline Scinax fuscomarginatus (Lutz, 1925) & 1,3 & $2,3,4,6$ & 2,3 & 1 \\
\hline S. fuscovarius (Lutz, 1925) & 1,3 & $2,3,4,6$ & 2,3 & 1 \\
\hline S. aff. similis & 1,3 & $2,3,4,6$ & 2,3 & 1 \\
\hline Trachycephalus typhonius (Linnaeus, 1758) & 1,3 & $2,3,6$ & 2 & 1 \\
\hline \multicolumn{5}{|l|}{ Leptodactylidae } \\
\hline Adenomera sp. & & $1,2,3,4,6$ & 2 & 30 \\
\hline Leptodactylus fuscus (Schneider, 1799) & $1,3,4$ & $2,3,4,6$ & 2,3 & 30 \\
\hline L. furnarius Sazima \& Bokermann, 1978 & 1,3 & $2,3,4,6$ & 2,3 & 30 \\
\hline L. sertanejo (Giaretta \& Costa, 2007) & 1,3 & $2,3,4,5,6$ & 6 & 30 \\
\hline L. labyrinthicus (Spix, 1824) & 1,3 & $2,3,4,6$ & 2,3 & 13 \\
\hline L. mystaceus (Spix, 1824) & 4 & 1 & 2,3 & 30 \\
\hline L. mystacinus (Burmeister, 1861) & 1,4 & $1,2,3,4,6$ & 2,3 & 30 \\
\hline L. cf. latrans & 1,4 & $2,3,4,6$ & 2,3 & 11 \\
\hline L. podicipinus (Cope, 1862) & 1 & $2,3,4,6$ & 2,3 & 13 \\
\hline L. syphax Bokermann, 1969 & 4 & 1 & 4 & 29 \\
\hline Physalaemus centralis Bokermann, 1962 & 1 & $2,3,4,6$ & 2 & 11 \\
\hline P. cuvieri Fitzinger, 1826 & 1,3 & $2,3,4,6$ & 2,3 & 11 \\
\hline P. marmoratus (Reinhardt \& Lütken, 1862) & & $2,3,4,6$ & 2,3 & 11 \\
\hline Eupemphix nattereri Steindachner, 1863 & 1 & $2,3,4,6$ & 2 & 11 \\
\hline Pseudopaludicola falcipes (Hensel, 1867) & 1 & $2,3,4,6$ & 5 & 1 \\
\hline P. mystacalis (Cope, 1887) & 1,3 & $2,3,4,6$ & 5 & 1 \\
\hline P.ternetzi Miranda-Ribeiro, 1937 & & 5 & 5 & 1 \\
\hline \multicolumn{5}{|l|}{ Microhylidae } \\
\hline Chiasmocleis albopunctata (Boettger, 1885) & 1,4 & $1,2,3,4$ & 2 & 1 \\
\hline Elachistocleis bicolor (Guérin-Méneville, 1838) & 1,4 & $2,3,4,6$ & 2,3 & 1 \\
\hline Elachistocleis cesarii (Miranda-Ribeiro, 1920) & 1,4 & $2,3,4,6$ & 2,3 & 1 \\
\hline Dematonotus muelleri (Boettger, 1885) & 4 & $2,3,4,6$ & 2,3 & 1 \\
\hline \multicolumn{5}{|l|}{ Odontophrynidae } \\
\hline Odontophrynus cultripes Reinhardt \& Lütken, 1862 & 1 & 1 & 2 & 1 \\
\hline Proceratophrys sp. (gr. cristiceps) & 3 & 1,5 & 4,6 & 2 \\
\hline
\end{tabular}

aproximadamente $50 \%(\bar{x} 48,18 \pm 7,95)$, sendo que a maior diferença percentual ocorre entre a CEC e CEF e a menor entre a FES e CEF (Tab. III). Apenas 22 das

95 espécies consideradas na análise contribuíram para a dissimilaridade na composição de espécies entre os quatro ecossistemas (Tab. IV). 
Tab. III. Valores das comparações par a par da ANOSIM indicando o valor do $\mathrm{R}$, e os valores de quebra de porcentagem de dissimilaridade (SIMPER) dos quatro ecossistemas/biomas analisados (CER, Cerrado; $\mathrm{CEC}$, transição entre Cerrado e a Caatinga; $\mathrm{CEF}$, transição entre o Cerrado e a Floresta Estacional Semidecidual; FES, Floresta Estacional Semidecidual).

\begin{tabular}{lccc}
\hline Grupos & $\mathrm{R}$ & $\mathrm{P}(\%)$ & SIMPER \\
\hline CEC e CEF & 2 & 1 & 61,19 \\
CER e CEC & 0,90 & 0,14 & 50,63 \\
CER e CEF & 0,35 & 0,13 & 47,04 \\
CER e FES & 0,29 & 0,02 & 43,22 \\
FES e CEC & 0,93 & 0,14 & 51,42 \\
FES e CEF & 0,02 & 0,50 & 35,01 \\
\hline
\end{tabular}

\section{DISCUSSÃO}

Ainda que a bacia do Rio Tijuco esteja descaracterizada pelo elevado grau de desmatamento que sofre há décadas, apresenta uma anurofauna diversa. O número de espécies registradas representa 20\% daquelas esperadas para o Cerrado brasileiro (VALDUJO et al., 2012), sendo uma das maiores listagens para uma localidade dentro do bioma (ver revisão em NomURA et al., 2012). Por ser a segunda maior ecorregião da América do Sul, o Cerrado abrange diferentes ecossistemas, apresentando grande heterogeneidade ambiental (KLINK et al., 2002), que é responsável pela maior disponibilidade de micro-hábitat acomodando mais espécies (PianKa, 1967; Huston, 1994; AfOnso \& EterovicK, 2007; BASTAZINI et al., 2007; RicHTERBoIX et al., 2007; VASCONCELOS et al., 2009; WERNER et al., 2009; HAMMER \& PARRIS, 2011; Silva et al., 2011, 2012).

Eventos históricos de dispersão de espécies, condições ambientais (relevo, altitude, clima e vegetação) e extinções locais são fatores que influenciam na composição e similaridade de espécies (JOHN-ALDER et al., 1988; Richter-Boix et al., 2007; Gotelli et al., 2009; Keller et al., 2009). No Brasil, estudos com distribuição têm revelado a influência dos domínios morfoclimáticos na composição de taxocenoses de anuros (CRump, 1971; Conte \& RossA-Feres, 2006, ZINA et al., 2007; VASCONCELOS et al., 2010), o que torna a distância geográfica e, consequentemente, as variáveis abióticas locais, mecanismos responsáveis pela estruturação dos padrões de composição e similaridade de espécies (INGER \& VorIS, 1993; PARris, 2004; VASCONCELOS et al., 2010).

O Cerrado tem como característica uma grande estacionalidade, com concentração de chuva nos meses de primavera e verão, enquanto que na estação seca os índices pluviométricos mensais são drasticamente reduzidos, podendo chegar a zero. Além disso, é formado por um mosaico complexo de savanas, campos e florestas secas cujo processo prevalente ecológico é o fogo, que influencia drasticamente na estrutura do ecossistema (EITEN, 1972; KAUFFMAN et al., 1994). Nestas condições, a sobrevivência dos organismos vai depender de características anatômicas, fisiológicas e comportamentais, favorecendo espécies mais tolerantes e adaptadas (ANDERSEN et al., 2005). No caso de anuros, parece ser refletido na baixa proporção de modos reprodutivos e a elevada representatividade de modos generalizados (modos 1 e 2) ou mais resistentes à dessecação e adaptados à insolação (11 e 30) pode ocupar habitats com chuvas imprevisíveis. Segundo Duellman \& Trueb (1986), a diversificação e especialização de modos tem mais relação com as características ambientais de determinada localidade do que com relações filogenéticas e a ausência de uma

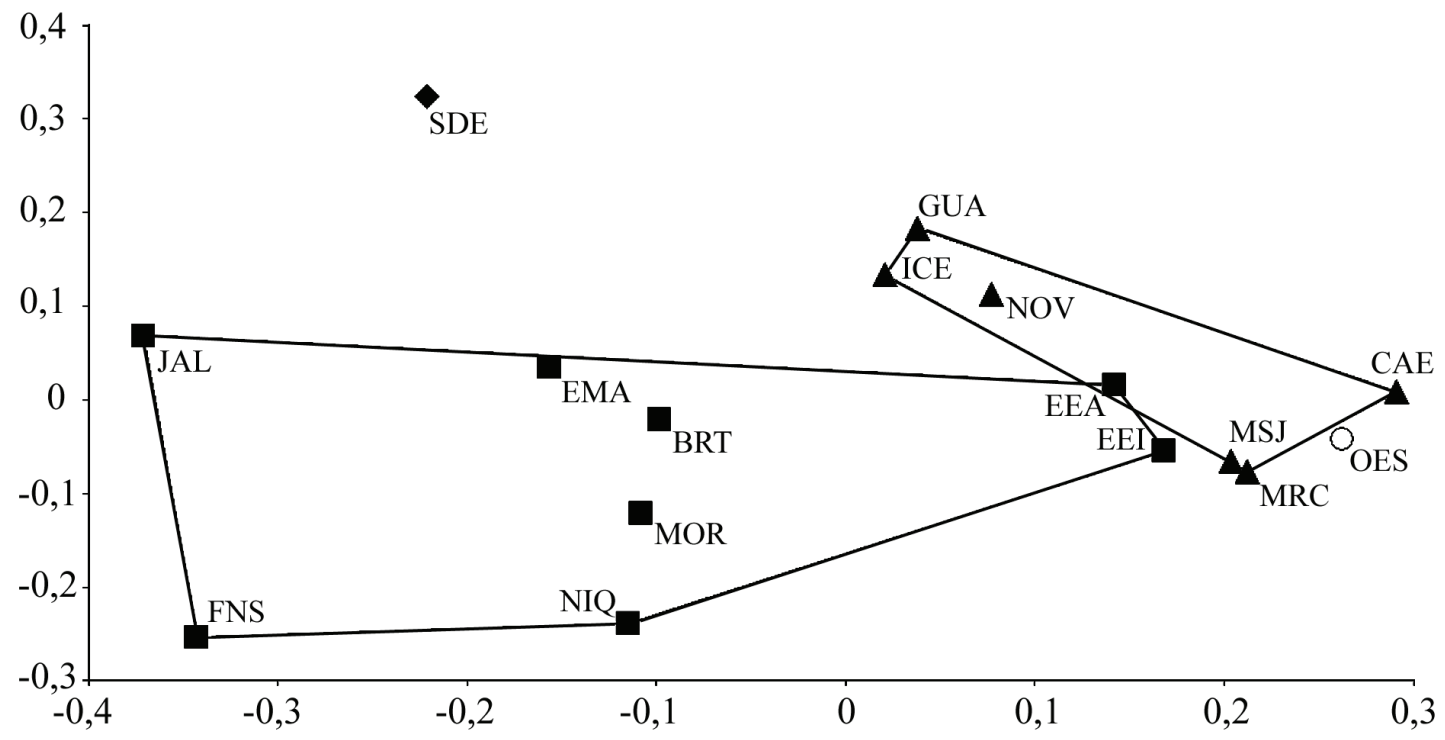

Fig. 2. Análise escalonada multidimensional não-métrica, resultante da composição de espécies de anfíbios registradas em 16 localidades. ( $\mathbf{\square}$, Cerrado: BRT, Bacia do Rio Tijuco; FNS, FLONA de Silvânia; EMA, PARNA de Emas; EEI, EE de Itirapina; EEA, EE de Assis; MOR, Morrinhos; NIQ, Município de Niquelândia; JAL, EE Serra Geral do Tocantins; O, Transição entre o Cerrado e a Floresta Estacional Semidecidual: OES, Fazenda Rio Claro; $\downarrow$, Transição entre o Cerrado e a Caatinga: SED, Município de São Desidério; $\boldsymbol{\Delta}$, Floresta Estacional Semidecidual: MSJ, Mata São José GUA, Município de Guararapes; NOV, Município de Nova Itapirema; CAE, EE dos Caetetus; ICE = Município de Icem; MRC = FLOE Edmundo Navarro de Andrade; PARNA = Parque Nacional, FLONA = Floresta Nacional, EE = estação ecológica; FLOE = Floresta Estadual 
Tab. IV. Contribuição das espécies à dissimilaridade média entre os possíveis pares de combinação dos quatro ecossistemas/biomas analisados (G): Cerrado (CER), transição entre Cerrado e a Caatinga (CEC), transição entre o Cerrado e a Floresta Estacional Semidecidual (CEF) e Floresta Estacional Semidecidual (FES)

\begin{tabular}{|c|c|c|c|}
\hline G & Espécies & Contribuição (\%) & Porcentagem Cumulativa \\
\hline \multirow{6}{*}{ 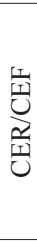 } & Vitreorana uranoscopa (Müller, 1924) & 3,29 & 3,29 \\
\hline & Dendropsophus anceps (Lutz, 1929) & 3,29 & 6,58 \\
\hline & D. elianeae (Napoli \& Caramaschi, 2000) & 3,29 & 9,87 \\
\hline & D. microps (Peters, 1872) & 3,29 & 13,17 \\
\hline & Hypsiboas caingua (Carrizo, 1991) & 3,29 & 16,46 \\
\hline & H. prasinus (Burmeister, 1856) & 3,29 & 19,75 \\
\hline \multirow{5}{*}{ 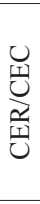 } & Rhinella cerradensis Maciel, Brandão, Campos \& Sebben, 2007 & 3,69 & 3,69 \\
\hline & Rhinella veredas (Brandão, Maciel \& Sebben, 2007) & 3,69 & 7,39 \\
\hline & D. melanargyreus (Cope, 1887$)$ & 3,69 & 11,08 \\
\hline & H. crepitans (Wied-Neuwied, 1824) & 3,69 & 14,77 \\
\hline & Physalaemus albifrons (Spix, 1824) & 3,69 & 18,46 \\
\hline \multirow{8}{*}{ 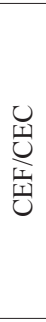 } & Vitreorana uranoscopa & 2,44 & 2,44 \\
\hline & Rhinella cerradensis & 2,44 & 4,88 \\
\hline & R. ornata (Spix, 1824) & 2,44 & 7,32 \\
\hline & R. mirandaribeiroi (Gallardo, 1965) & 2,44 & 9,76 \\
\hline & R. veredas & 2,44 & 12,20 \\
\hline & Odontophrynus americanus (Duméril \& Bibron, 1841) & 2,44 & 14,63 \\
\hline & Proceratophrys moratoi (Jim \& Caramaschi, 1980) & 2,44 & 17,07 \\
\hline & Aplastodiscus perviridis Lutz, 1950 & 2,44 & 19,51 \\
\hline \multirow{7}{*}{ 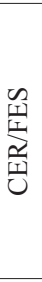 } & D. elianeae & 2,96 & 2,96 \\
\hline & D. sanborni (Schmidt, 1944) & 2,92 & 5,88 \\
\hline & Physalaemus marmoratus (Reinhardt \& Lütken, 1862) & 2,86 & 8,73 \\
\hline & Barycholos ternetzi & 2,73 & 11,46 \\
\hline & Phyllomedusa azurea Cope 1862 & 2,73 & 14,19 \\
\hline & D. cruzi & 2,70 & 16,9 \\
\hline & L. mystaceus & 2,47 & 19,36 \\
\hline \multirow{4}{*}{ 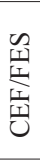 } & Proceratophrys moratoi & 4,47 & 4,47 \\
\hline & Aplastodiscus perviridis & 4,47 & 8,95 \\
\hline & D. anceps & 4,47 & 13,42 \\
\hline & D. jimi & 4,47 & 17,90 \\
\hline \multirow{5}{*}{ 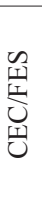 } & Rhinella cerradensis & 3,68 & 3,68 \\
\hline & R. mirandaribeiroi & 3,68 & 7,37 \\
\hline & $R$. veredas & 3,68 & 11,05 \\
\hline & D. melanargyreus & 3,68 & 14,73 \\
\hline & H. crepitans & 3,68 & 18,42 \\
\hline
\end{tabular}

maior variedade de micro-hábitats úmidos, impede uma alta proporção de espécies que apresentem modos reprodutivos especializados. Não obstante, a elevada representatividade de modos reprodutivos generalizados pode ocorrer em decorrência da menor heterogeneidade ambiental dessas localidades, principalmente decorrente da maciça paisagem agropastoril bem como uma sazonalidade acentuada, quando comparadas com a Floresta Atlântica, tal como ocorre em outras regiões de áreas abertas alteradas: 0,2 (4 modos/21 espécies), em Rio Claro (Toledo et al., 2003); 0,15 (4 modos/27 espécies) em Nova Itapirema (VASCONCELOS \& RossAFERES, 2005).

Das 40 espécies registradas, mais da metade fazem o depósito de seus ovos e têm o desenvolvimento dos seus girinos em ambientes lênticos (modo 1, conforme HadDAD \& Prado, 2005). Ovos depositados diretamente na água em ambientes lênticos e totalmente dependentes da água para todas as fases do desenvolvimento larval é o modo mais comum e filogeneticamente difundido e generalizado (Duellman \& TRUEB, 1986). Os modos que envolvem ninhos de espuma construídos pelos representantes da família Leptodactylidae (modos 11, 13 e 30), também são bem difundidos entre as espécies do cerrado, pois provém uma excelente proteção contra dessecação (Duellman \& Trueb, 1986; Downie, 1988; PRADO et al., 2002).

A dependência da água pelos anfíbios da bacia do Rio Tijuco demonstra a importância destes tipos de ambientes para a manutenção das espécies locais, onde todos os ambientes lênticos e lóticos, bem como a estrutura física de seu entorno, são de interesse para a preservação da anurofauna. Neste sentido, vale ressaltar que mais de $50 \%$ das espécies de anfíbios da região foram registradas em locais alterados por perturbações humanas, como os açudes temporários que são utilizados para irrigação de lavouras ou fonte d'água para gado, tal como observado por GiaReTta et al. (2008). As paisagens com maior oferta de corpos d'água podem ocasionar maior chance de encontro destes novos habitats para reprodução 
(Hazell et al., 2001; Nomura et al., 2012). Além disso, como a maioria dos corpos d'água artificiais da região é temporário, inviabiliza a existência de taxocenoses de peixes e favorece a ocupação pelos anuros, uma vez que peixes afetam a diversidade de presas - no caso girinos -, produzindo padrões de distribuição e diversidade distintos entre habitats com e sem estes predadores (Heyer et al., 1975; Hero et al., 2001; EgAn \& PATON, 2004). Apesar da imensa alteração ambiental causada por pastagens (LONGO et al., 1999; KAIMOWITZ, 2002) é um tipo de uso de solo diferente de outras culturas que apresentam intensa expansão nos domínios do Cerrado como o cultivo da cana-de-açúcar (NASSAR et al., 2008), uma monocultura de crescimento rápido e ciclo curto, havendo grande demanda de água, o que frequentemente leva à exaustão de nascentes e mananciais (GUARNIERI \& JANNUZZI, 1992), além alteração da diversidade animal, como por exemplo taxocenoses de aves (PIrATElli et al., 2005).

Apesar da maioria das espécies serem caracterizadas como de área aberta e potenciais colonizadores de habitat antrópicos, há uma relação positiva da abundância e riqueza de espécies de anfíbios com a área de cobertura florestal nativa (HECNAR \& M'Closkey, 1997; VAllan, 2000; Guerry \& Hunter, 2002), na qual os fragmentos florestais exercem influência na ocupação dos corpos d'água localizados em área aberta (LAAN \& VerboOM, 1990). Ademais, os fragmentos florestais são de extrema importância para a dispersão dos juvenis de anuros, que evitam as áreas abertas devido ao alto risco de dessecação (ROTHERMEL \& Semlitsch, 2002; Rothermel, 2004) e podem servir de refúgios para os adultos algumas espécies (Weyrauch \& GrubB, 2004). Nos remanescentes florestais estudados, grande quantidade de jovens de Leptodactylus mystacinus, L. mystaceus e adultos de Dendropsophus cf. melanargyreus e Chiasmocleis albopunctata foram capturados em armadilhas de interceptação e queda e/ou encontrados pela busca ativa no interior de remanescentes florestais, além das espécies registradas em atividade de vocalização exclusivamente no interior dos remanescentes florestais, caso de Odontoprhynus cultripes, Proceratophrys sp. (gr. cristiceps) e Barycholos ternetzi. Desse modo, as espécies registradas certamente não estarão distribuídas ao longo de toda a área, podendo ocorrer substituições e até mesmo a ausência de algumas delas em alguns pontos específicos.

Toda região do Triângulo Mineiro foi intensamente devastadas desde o século XIX, em razão do solo oferecer condições favoráveis para a pecuária (GoBBI et al., 2008). Certamente essas modificações interferiram e continuam a influenciar a dinâmica das populações e a distribuição das espécies, uma vez que as principais causas da alteração na riqueza e abundância de anfíbios é a supressão da cobertura vegetal (AICHINGER, 1991; Tocher et al., 1997; Marsh \& Pearman, 1997). Porém não se sabe o real impacto sobre a taxocenose de anuros local porque que não existem dados bionômicos pretéritos à alteração ambiental.

Assim, numa perspectiva de conservação, o que se pode afirmar é que a expressiva riqueza de anuros é atribuída à complexidade da paisagem, alternando áreas abertas com corpos d'água artificiais que possibilitam o sucesso reprodutivo de diversas espécies de anuros (VASCONCElOS \& RosSa-Feres, 2005; Nomura et al., 2012), associados a remanescentes florestais que servem para manutenção de espécies estritamente florestais quanto refúgio para espécies de áreas abertas (Weyrauch \& Grubb, 2004; Silva \& Rossa-Feres 2007).

Agradecimentos. À Darlene da Silva Gonçalves e Gledson Vigiano Bianconi pela leitura do manuscrito. À Coordenadoria de Aperfeiçoamento de Pessoal de Nível Superior (CAPES) pela bolsa concedida a C.E.C. (PRODOC n $\left.{ }^{\circ} 18-32 / 2010\right)$.

\section{REFERÊNCIAS BIBLIOGRÁFICAS}

Afonso, L. G. \& Eterovick, P. C. 2007. Microhabitat choice and differential use by anurans in forest streams in southeastern Brazil. Journal of Natural History 41:937-948.

Aichinger, M. 1991. Faunal deficit of anurans in tropical farmland of Amazonian, Peru. Alytes 9:23-32.

Andersen, A. N.; Cook, G. D.; Corbett, L. K.; Douglas, M. M.; Eager, R. W.; Russel-Smith, J.; Setterfield, S. A.; Williams, R. J. \& WOINARSKI, J. C. Z. 2005. Fire frequency and biodiversity conservation in Australian tropical savannas: implications from the Kapalga fire experiment. Austral Ecology 30:155-167.

Bastazini, C. V.; Munduruca, J. F. V.; Rocha, P. L. B. \& Napoli, M. F. 2007. Which environmental variables better explain changes in anuran community composition? A case study in the Restinga of Mata de São João, Bahia, Brazil. Herpetologica 63(4):459-471.

Bastos, R. P. 2007. Anfíbios do Cerrado. In: Nascimento, L. B. \& Oliveira, M. E. eds. Herpetologia no Brasil II. Belo Horizonte, Sociedade Brasileira de Herpetologia. p.87-100.

Bastos, R. P.; Motta, J. A. O.; Lima, L. P. \& Guimarães, L. D. 2003 Anfíbios da Floresta Nacional de Silvânia, estado de Goiás. Goiânia, Stylo Gráfica e Editora. 82p.

Bernarde, P. S. \& Kokubum, M. N. C. 1999. Anurofauna do município de Guararapes, estado de São Paulo, Brasil (Amphibia, Anura). Acta Biológica Leopoldensia 21(1):89-97.

Borges, F. J. A. \& Juliano, R. F. 2007. Distribuição espacial e temporal de uma comunidade de anuros do município de Morrinhos, Goiás, Brasil (Amphibia: Anura). Neotropical Biology and Conservation 2(1):21-27.

Brasileiro, C. A.; Sawaya, R. J.; Kiefer, M. C. \& Martins, M. 2005. Amphibians of an Open Cerrado Fragment in Southeastern Brazil. Biota Neotropica 5(2). Disponível em: $<$ http://dx.doi.org/10.1590/ S1676-06032005000300006>. Acesso em: 01/03/2010.

Brassaloti, R. A.; Rossa-Feres, D. C. \& Bertoluci, J. 2010 Anurofauna da Floresta Estacional Semidecidual da Estação Ecológica dos Caetetus, sudeste do Brasil. Biota Neotropica 10(1). Diponível em: <http://www.biotaneotropica.org.br/v10n1/ pt/abstract?inventory+bn01810012010>. Acesso em 01/03/2011.

CLARKE, K. R. 1993. Non-parametric multivariate analysis of changes in community structure. Australian Journal of Ecology 18:117-143.

Clarke, K. R. \& Gorley, R. N. 2006. Software PRIMER v6. Plymouth, PRIMER-E.

Clarke, K. R. \& Warwick. R. M. 1994. Chance in marine communities: an approach to statistical analysis and interpretation. Bournemouth, Bourne Press. 128p.

Colli, G. R.; Bastos, R. P. \& Araujo, A. F. B. 2002. The character and dynamics of the Cerrado herpetofauna. In: Oliveira, P. S. \& Marquis, R. J. eds. The Cerrados of Brazil. Ecology and natural history of a Neotropical savanna. New York, Columbia University Press. p.223-241. 
Conte, C. E. \& Rossa-Feres, D. C. 2006. Diversidade e ocorrência temporal da anurofauna (Amphibia, Anura) em São José dos Pinhais, Paraná, Brasil. Revista Brasileira de Zoologia 23(2):162-175.

CoRn, P. S. 1994. Straight-Line Drift Fences and Pitfall Traps. In: Heyer, W. R.; Donnelly, M. A.; McDiarmid, R. W.; HayeK, L. A. C. \& Foster, M. S. eds. Measuring and Monitoring Biological Diversity - Standard Methods for amphibians. Washington D.C., Smithsonian Institution Press. p.109-118.

Coutinho, L. M. 1978. O conceito de Cerrado. Revista Brasileira de Botânica 1:17-23.

CrumP, M. L. 1971. Quantitative analysis of the ecological distribution of a tropical herpetofauna. Occasional Papers of the Museum of Natural History 3:1-62.

Diniz-Filho, J. A. F.; Bini, L. M.; Vieira, C. M.; Souza, M. C.; Bastos, R. P.; Brandão, D. \& Oliveira, L. G. 2004a. Spatial patterns in species richness and priority areas for conservation of anurans in the Cerrado region, Central Brazil. Amphibia-Reptilia 25:63-75

Diniz-Filho, J. A. F.; Bini, L. M.; Bastos, R. P.; Vieira, C. M.; Souza, M. C.; Motta, J. A. O.; Pombal JR., J. P. \& Peixoto, J. C. 2004b. Anurans from a local assemblage in Central Brazil: linking local processes with macroecological patterns. Brazilian Journal of Biology 64:41-52.

DownIE, J. R. 1988. Functions of the foam in the foamnesting leptodactylid Physalaemus pustulosus. Herpetological Journal 1:302-307.

Duellman, W. E. \& Trueb, L. 1986. Biology of amphibians. New York, McGraw-Hill Book Company. 670p.

Egan R. S. \& PATON, P. W. C. 2004. Within-pond parameters affecting oviposition by wood frogs and spotted salamanders. Wetlands 24(1):1-13.

Eiten, G. 1972. The Cerrado vegetation of Brazil. The Botanical Review 38:201-341.

Eterovick, P. C. \& Sazima, I. 2004. Anfíbios da Serra do Cipó, Minas Gerais. Belo Horizonte, PUC Minas. 152p.

Frost, D. R. 2013. Amphibian species of the world: an online reference. Version 5.5 New York. Disponivel em: <http:/ research.amnh.org/vz/herpetology/amphibia/AmericanMuseumof NaturalHistory.> Acesso em 10.04.2013

Giaretta, A. A.; Menin, M.; Facure, K. G.; Kokubum, M. N. C. \& OliveIRA-FILHO, J. C. 2008. Species richness, relative abundance, and habitat of reproduction of terrestrial frogs in the Triângulo Mineiro region, Cerrado Biome, southeastern Brazil. Iheringia, Série Zoologia 98(2):181-188.

Gobbi, G. A. F; Torres, J. L. R. \& Fabian, A. J. 2008. Diagnóstico ambiental da microbacia do córrego do melo em Uberaba (MG). Caminhos de Geografia 9(26):206-223

Gotelli, N. J.; Anderson, M. J. \& Arita, H. T. 2009. Patterns and causes of species richness: a general simulation model for macroecology. Ecology Letters 12:873-886.

GuARnieri, L. C. \& Jannuzzi, R. M. 1992. Proálcool: Impactos Ambientais. Revista Brasileira de Energia 2(2):6-10.

GUERRY, A. D. \& Hunter JR, M. L. 2002. Amphibian distributions in a landscape of forests and agriculture: an examination of landscape composition and configuration. Conservation Biology 16:745754

Haddad, C. F. B. \& Prado, C. P. A. 2005. Reproductive modes in frogs and their unexpected diversity in the Atlantic Forest of Brazil. Bioscience 55:207-217.

Haddad, C. F. B.; Andrade, G. V. \& Cardoso, A. J. 1988. Anfíbios Anuros no Parque Nacional da Serra da Canastra, Estado de Minas Gerais. Brasil Florestal 64:9-20.

HAMER, A. J. \& PARRIS, K. M. 2011. Local and landscape determinants of amphibian communities in urban ponds. Ecological Applications 21:378-390.

Hammer, Ø; Harper, D. A. T. \& RYAN, P. D. 2001. PAST: Paleontological Statistics software package for education and data analysis. Palaeontologia Electronica 4(1):1-9.

Hazell, D.; Cunnningham, R.; Lindenmayer, D.; Mackey, B. \& Osborne, W. 2001. Use of farm dams as frog habitat in an Australian agricultural landscape: factors affecting species richness and distribution. Biological Conservation 102:155-169.

Hecnar, S. J. \& M'Closkey, R. T. 1997. The effects of predatory fish on amphibian species richness and distribution. Biological
Conservation 79:123-131.

Hero, J. M.; Magnusson, W. E.; Rocha, C. F. D. \& Catterall, C. P. 2001. Antipredator defenses influence the distribution of amphibian prey species in the central Amazon rain forest Biotropica 33(1):131-141.

Heyer, W. R.; McDiarmid, R.W. \& Weigmann, D. L. 1975. Tadpoles, predation and pond habitats in the tropics. Biotropica 7:100-111.

Huston, M. A. 1994. Biological diversity - the coexistence of species on changing landascapes. New York, Cambridge Univesity Press. 778p.

INGER, R. F. \& Voris, H. K. 1993. A Comparison of Amphibian Communities through Time and From Place to Placein Bornean Forests. Journal of Tropical Ecology 9(4):409-433.

John-Alder, H. B.; Morin, P. J. \& Lawler, S. 1988. Thermal physiology, phenology, and distribution of tree frogs. The American Naturalist 132(4):506-520.

Kaimowitz, D. 2002. Las causas subjacentes de la deforestación en el trópico. In: Guariguata, M. R. \& Kattan, G. H. eds. Ecología y Conservación de Bosques Neotropicales. México, Ediciones Lur. 597p.

Kauffman, J. B.; Cummings, D. L. \& Ward, D. E. 1994. Relationships of fire, biomass and nutrient dynamics along a vegetation gradient in the Brazilian Cerrado. Journal of Ecology 82:519-531

Keller, A.; Rödel, M. O.; Linsenmair, K. E. \& Grafe, T. U. 2009. The importance of environmental heterogeneity for species diversity and assemblage structure in Bornean stream frogs. Journal of Animal Ecology 78(2):305-314

KlinK, C. A. \& Machado, R. B. 2005. Conservation of Brazilian Cerrado. Conservation Biology 19:707-713.

Klink, C. A.; Miranda, H.; GonZÁles, I. \& Vicentinni, K. 2002. O Bioma Cerrado - Site 3. In: Os sites e o programa brasileiro de pesquisas ecológicas de longa duração. 1ed. Porto Alegre, Fundação da Universidade Federal do Rio Grande do Sul, Universidade Federal de Minas Gerais. v. 1. p.51-68.

KopP, K.; SignORELli, L. \& Bastos, R. P. 2010. Distribuição temporal e diversidade de modos reprodutivos de anfíbios anuros no Parque Nacional das Emas e entorno, estado de Goiás, Brasil. Iheringia, Série Zoologia 100(3):192-200.

LaAn, R. \& Verboom, B. 1990. Effects of pool size and isolation on amphibian communities. Biological Conservation 54:251-262.

Legendre, P. \& Legendre, L. 1998. Numerical Ecology. Amsterdam, Elsevier. 1006p.

Longo, R. M.; EsPíndola, C. R. \& Ribeiro, A. I. 1999. Modificações na estabilidade de agregados no solo decorrentes da introducão de pastagens em áreas de Cerrado e Floresta Amazônica. Revista Brasileira de Engenharia Agrícola e Ambiental 3(3):276-280.

Maffei, F.; Ubaid, F. K. \& Jim, J. 2011. Anfíbios: Fazenda Rio Claro, Lencóis Paulista, SP, Brasil. Bauru, Canal 6 editora. 126p.

Manly, B. J. F. 2008. Métodos Estatísticos Multivariados. 3ed. Porto Alegre, Bookman. 229p

Marsh, D. M. \& Pearman, P. B. 1997. Effects of habitat fragmentation on the abundance of two species of Leptodactylid frogs in an Andean montane Forest. Conservation Biology 11:1323-1328.

MMA - Ministério do Meio AMBIEnte. 2002. Avaliação e identificação de áreas e ações prioritárias para a conservação, utilização sustentável e repartição dos benefícios da biodiversidade nos biomas brasileiros. Brasília, Ministério do Meio Ambiente. $404 \mathrm{p}$

Myers, N.; Mittermeier, R. A.; Mittermeier, C. G.; Fonseca, G. A. B. \& Kent, J. 2000. Biodiversity hotspots for conservation priorities. Nature 403:853-858.

Nassar, A. M.; RudorfF, L. B. A.; Aguiar, D. A.; Bacchi, M. R. P. \& ADAMI, M. 2008. Prospects of the sugarcane expansion in Brazil impacts on direct and indirect land use changes. In: ZUURBIER, P. \& Vooren, J. V. eds. Sugarcane Ethanol: contributions to climate change mitigation and the environment. 1ed. The Netherlands, Wageningen Academic Press. 255p.

Nomura, F.; Maciel, N. M.; Pereira, E. B. \& Bastos, R. P. 2012. Diversidade de anuros (Amphibia) em áreas recuperadas de atividade mineradora e de plantio de Eucalyptus urophyla, no Brasil Central. Bioscience Journal 28(2):312-324.

PARris, K. M. 2004. Environmental and spatial variables influence the composition of frog assemblages in subtropical eastern Australia. Ecography 27:392-400

PHILLIPS, K. 1990. Where have all the frogs and toads gone? Bioscience 40:422-424. 
PianKA, E. R. 1967. On lizard species diversity: North American flatland deserts. Ecology 48:333-351

Piratelli, A.; Andrade, V. A. \& Lima-Filho, M. 2005. Aves de fragmentos florestais em área de cultivo de cana-de-açúcar no sudeste do Brasil, Iheringia, Série Zoologia 95(2):217-222.

Prado, C. P. A.; Uetanabaro, M. \& Haddad, C. F. B. 2002. Description of a new reproductive mode in Leptodactylus (Anura, Leptodactylidae), with a review of the reproductive specialization toward terrestriality in the genus. Copeia 2002(4):1128-1133.

Reaser, J. K. 2000. Amphibian declines: an issue overview. Washington, Federal Taskforce on amphibian declines and deformities. $32 \mathrm{p}$.

R Development Core Team. 2011. R: A language and environment for statistical computing, reference index version 2.13.1. Vienna, R Foundation for Statistical Computing. Disponível em: $<$ http://www.Rproject.org >. Acesso em 01.02.2012.

Ribeiro-Júnior, J. W. \& Bertoluci, J. 2009. Anuros do cerrado da Estação Ecológica e da Floresta Estadual de Assis, sudeste do Brasil. Biota Neotropica 9(1). Disponível em: <http:/ dx.doi.org/10.1590/S1676-06032009000100020>. Acesso em 04.04.2013.

Richter-Boix, A.; Llorente, G. A. \& Montori, A. 2007. Structure and dynamics of an amphibian metacommunity in two regions. Journal of Animal Ecology 76:607-618

Rocha, C. F. D. 1994. Introdução a Ecologia de Lagartos Brasileiros. In: Nascimento, L. B.; Bernardes, A. T. \& Cotta, G. A. eds. Herpetologia no Brasil, 1. Belo Horizonte, PUC-Minas Gerais. p.39-57.

Rothermel, B. B. 2004. Migratory success of juveniles: a potential constraint on connectivity for pond-breeding amphibians. Ecological Applications 14(5): 1535-1546.

Rothermel, B. B. \& Semlitsch, R. D. 2002. An experimental investigation of landscape resistance of forest versus old-field habitats to emigrating juvenile amphibians. Conservation Biology 16:1324-1332.

SANTOS, L. \& BACCARO, C. A. D. 2004. Caracterização geomorfológica da bacia do rio Tijuco. Caminhos da Geografia 5(11). Disponível em: <http://www.caminhosdegeografia.ig.ufu.br/include/getdoc. php?id $=306 \&$ article $=80 \&$ mode $=$ pdf $>$. Acesso em: 17.01.2013.

SCOTT JR., N. J. \& WoODWARD, B. D. 1994. Surveys at breeding sites. In: Heyer, W. R.; Donnelly, M. A.; McDiarmid, R. W.; HayeK, L. A. C. \& Foster, M. S. eds. Measuring and Monitoring Biological Diversity - Standard Methods for amphibians. Washington, Smithsonian Institution Press. p. 118-125.

Silva, F. R. \& Rossa-Feres, D. C. 2007. The use of forest fragments by open-area anurans (Amphibia) in northwestern São Paulo State, Brazil. Biota Neotropica 7:141-148.

Silva, F. R.; Candeira, C. P. \& Rossa-Feres, D. C. 2012. Dependence of anuran diversity on environmental descriptors in farmland ponds. Biodiversity Conservation 21:1411-1424.

Silva, F. R.; GibBs, J. P. \& Rossa-Feres, D. C. 2011. Breeding Habitat and Landscape Correlates of Frog Diversity and Abundance in a Tropical Agricultural Landscape. Wetlands 31:1079-1087.

Silveira, A. L. 2006. Anfibios do município de João Pinheiro, uma área de Cerrado no noroeste de Minas Gerais. Arquivos do Museu Nacional 64(2):131-139.

Strüssmann, C. 2000. Herpetofauna. In: Alho, C. J. R. coord. Fauna Silvestre da região do Rio Manso, MT. Brasília, IBAMA, ELETRONORTE. p.153-189.

Tocher, M. D.; GASCON, G. \& Zimmerman, B. L. 1997. Fragmentation effects on a Central Amazonian frog community: a ten-year study. In: Laurence, W. F. \& BierregaArd, R. O. eds. Tropical forest remnants: ecology, management, and conservation of fragmented communities. London, The University of Chicago Press. p.124-127.

Toledo, L. F; Zina, J. \& HADDAD, C. F. B. 2003. Distribuição espacial e temporal de uma comunidade de anfíbios anuros do Município de Rio Claro, São Paulo, Brasil. Holos Environment 3(2):136-149.

Valdujo, P. H.; Recoder, R. S.; Vasconcellos, M. M. \& Portella, A. S. 2009. Amphibia, Anura, São Desidério, western Bahia uplands, northeastern Brazil. Check List 5(4):903-911.

Valdujo, P. H.; Silvano, D. L.; Colli, G. \& Martins, M. 2012. Anuran Species Composition and Distribution Patterns in Brazilian Cerrado, a Neotropical Hotspot. South American Journal of Herpetology 7:63-78.

Valdujo, P. H.; Camacho, A.; Recoder, R. S.; Teixeira-Junior, M.; Ghellere, J. M. B.; Mott, T.; Nunes, P. S.; Nogueira, C. \& Rodrigues, M. T. 2011. Anfíbios da Estação Ecológica Serra Geral do Tocantins, região do Jalapão, Estados do Tocantins e Bahia. Biota Neotropica 11(1):251-262.

VALLAN, D. 2000. Influence of forest fragmentation on amphibian diversity in the nature reserve of Ambohitantely, highland Madagascar. Biological Conservation 96:31-43.

Vasconcelos, T. S. \& Rossa-Feres, D. C. 2005. Diversidade, distribuição espacial e temporal de anfíbios anuros (Amphibia Anura) na região noroeste do estado de São Paulo, Brasil. Biota Neotropica 5(2). Disponível em: <http://dx.doi.org/10.1590/ S1676-06032005000300010> Acesso em: 01.04.2008.

Vasconcelos, T. S.; Santos, T. G.; Rossa-Feres, D. C. \& Haddad, C. F. B. 2009. Influence of the environmental heterogeneity of breeding ponds on anuran assemblages from southeastern Brazil. Canadian Journal of Zoology 87:699-707.

Vasconcelos, T. S.; Santos, T. G.; Haddad, C. F. B. \& Rossa-Feres, D. C. 2010. Climatic variables and altitude as predictors of anuran species richness and number of reproductive modes in Brazil. Journal of Tropical Ecology 26:423-432.

WeLLS, K. D. 2007. The ecology and behavior of amphibians. Chicago University Press. 1148p.

Werner, E. E.; Relyea, R. A.; Yurewicz, K. L.; Skelly, D. K. \& DAVIS, C. J. 2009. Comparative landscape dynamics of two anuran species: climate-driven interaction of local and regional processes. Ecological Monographs 79:503-521.

Weyrauch, S. L. \& GrubB JR., T. C. 2004. Patch and landscape characteristics associated with the distribution of woodland amphibians in an agricultural fragmented landscape: an informationtheoretic approach. Biological Conservation 115:443-450.

Young, B.; Stuart, S. N.; Chanson, J. S.; Cox, N. A. \& Boucher, T. M. 2004. Disappearing jewels: The status of New World amphibians. Arlington, NatureServe, EUA. 54p.

Zimmerman, B. L. 1994. Audio Strip Tronsects. In: Heyer, W. R.; Donnelly, M. A.; McDiarmid, R. W.; HayeK, L. A. C. \& Foster, M. S. eds. Measuring and Monitoring Biological Diversity Standard Methods for amphibians. Washington, Smithsonian Institution Press. p.92-97.

Zina, J.; Ennser, J.; Pinheiro, S. C. P.; Haddad, C. F. B \& Toledo, L. F. 2007. Taxocenose de anuros de uma mata semidecídua do interior do Estado de São Paulo e comparações com outras taxocenoses do Estado, sudeste do Brasil. Biota Neotropica 7(2). Disponível em: <http://www.biotaneotropica.org.br/v7n2/pt/ abstract?article+bn00607022007> Acesso em 01.10.2012. 